Research paper

\title{
Identification of mutations in Colombian patients affected with Fabry disease
}

\author{
Alfredo Uribe ${ }^{\mathrm{a}, *, 1}$, Heidi Eliana Mateus ${ }^{\mathrm{b}, 1}$, Juan Carlos Prieto ${ }^{\mathrm{c}}$, Maria Fernanda Palacios ${ }^{\mathrm{c}}$, \\ Sandra Yaneth Ospina ${ }^{\mathrm{b}}$, Gabriela Pasqualim ${ }^{\mathrm{d}}$, Ursula da Silveira Matte ${ }^{\mathrm{d}, \mathrm{e}}$, Roberto Giugliani ${ }^{\mathrm{d}, \mathrm{e}}$ \\ a Centro de Investigaciones en Bioquímica (CIBI), Departamento de Ciencias Biológicas, Universidad de Los Andes, Carrera 1 No. $18^{\circ}$ - 12, Bogotá, Colombia \\ b Unidad de Genética, Escuela de Medicina y Ciencias de la Salud, Universidad del Rosario, Carrera 24 N 63C-69, Bogotá, Colombia \\ c Instituto de Genética, Facultad de Medicina, Universidad Javeriana, Carrera 7 No. 40-62 Ed. 32, Bogotá, Colombia \\ ' Centro de Terapia Gênica, Centro de Pesquisa Experimental, Hospital de Clínicas de Porto Alegre, Rua Ramiro Barcelos, 2350, Porto Alegre, RS 90035-003, Brazil \\ e Serviço de Genética Médica, Hospital de Clínicas de Porto Alegre, Rua Ramiro Barcelos, 2350, Porto Alegre, RS 90035-003, Brazil
}

\section{A R T I C L E I N F O}

\section{Article history:}

Received 27 November 2014

Received in revised form 5 August 2015

Accepted 10 August 2015

Available online 18 August 2015

\section{Keywords:}

$\alpha$-galactosidase A

Mutations

GLA gene

Lysosomal disorder

\begin{abstract}
A B S T R A C T
Fabry Disease (FD) is an X-linked inborn error of glycosphingolipid catabolism, caused by a deficiency of the lisosomal $\alpha$-galactosidase A (AGAL). The disorder leads to a vascular disease secondary to the involvement of kidney, heart and the central nervous system. The mutation analysis is a valuable tool for diagnosis and genetic counseling. Although more than 600 mutations have been identified, most mutations are private. Our objective was to describe the analysis of nine Colombian patients with Fabry disease by automated sequencing of the seven exons of the GLA gene. Two novel mutations were identified in two patients affected with the classical subtype of FD, in addition to other 6 mutations previously reported. The present study confirms the heterogeneity of mutations in Fabry disease and the importance of molecular analysis for genetic counseling, female heterozygotes detection as well as therapeutic decisions.
\end{abstract}

(C) 2015 Elsevier B.V. All rights reserved.

\section{Introduction}

Fabry disease (FD; OMIM: 301500) is an X-linked lysosomal storage disorder caused by the deficiency of the enzyme alpha-galactosidase $\mathrm{A}$ (AGAL, EC 3.2.1.22) (Brady, 1967). This enzymatic defect leads to the systemic accumulation of glycosphingolipids (mainly globotriaosylceramide -GL3-) in blood vessels from the skin, kidney, heart and brain (Desnick et al., 2003).

Today two main FD subtypes have been described. The first one, or "classical phenotype", affects males who have a markedly reduced AGAL activity. In these patients the onset of disease occurs in childhood or adolescence and is characterized by acroparesthesias, angiokeratomas, corneal opacities, hypohydrosis and progressive vasculopathy of the kidney, heart, and central nervous system (Desnick and Brady, 2004). The second phenotype, or "milder", has been described in patients with a higher residual AGAL activity and a later onset characterized by cardiac and renal symptoms (Nakao et al., 2003, 1995; Terryn et al., 2013).

Abbreviations: FD, Fabry disease; AGAL, alpha-galactosidase A; GLA, alpha-galactosidase A gene; GL3, globotriaosylceramide; DBS, dried blood sample.

* Corresponding author.

E-mail addresses: jeuribe@uniandes.edu.co (A. Uribe), heidi.mateus@urosario.edu.co (H.E. Mateus)

${ }^{1}$ A. Uribe and H. E. Mateus contributed equally to this work.
Due to the late appearance of symptoms in patients with milder FD, the prevalence of this phenotype seems to be higher in relation to patients with classical FD, whose incidence has been estimated to be between 1:40.000 to 1:117.000 male births, approximately (Lin et al., 2009; Meikle et al., 2006; Spada et al., 2006).

Patients with FD can be diagnosed by evaluating AGAL activity in plasma or white blood cells, however this analysis often fails to distinguish between Fabry heterozygotes with high residual AGAL activity and normal individuals. For this reason, mutation analyses are required to detect female heterozygotes, define genotype/phenotype correlations, and to make an accurate prenatal diagnosis and take therapeutic decisions (Yoshimitsu et al., 2011; Lukas et al., 2013).

The genomic sequence of the GLA gene is $12 \mathrm{~kb}$ in length and contains 7 exons (OMIM: 300644). To date, more than 600 mutations have been described including missense, nonsense and splice-site mutations, as well as gene rearrangements (Ashley et al., 2001; Schirinzi et al., 2008; Shabbeer et al., 2006). Most of the described mutations are private (with some few exceptions found in several unrelated subjects) and are usually associated with modifications in CPG dinucleotides, known hotspots for the disease (Barker et al., 1984; Cooper and Youssoufian, 1988).

In this study, we describe the first clinical and genetic analysis of nine Colombian FD patients. Direct sequencing of the complete GLA open reading frame revealed eight mutations, two new and six previously reported. Patients with the classical phenotype of FD were identified, 
thereby providing additional information about the genotypephenotype correlation.

\section{Materials and methods}

\subsection{Patients}

Nine FD Colombian patients were included in this study. All procedures were followed in accordance with the ethical standards of the responsible committee on human experimentation (institutional and national) and with the Helsinki Declaration of 1975, as revised in 2000 (5). All participants completed a consent form for diagnostic testing approved by the Institutional Ethics Committee of each participating institution.

\subsection{AGAL activity assays}

AGAL enzymatic activity was first determined in dried blood spot (DBS) samples (as previously described by Chamoles et al., 2001; Civallero et al., 2006;Uribe and Giugliani, 2013) and the results were compared with the reference values reported for the Colombian population (range: $2.0-21.8 \mathrm{nmol} / \mathrm{ml} / \mathrm{h}$; Uribe and Giugliani, 2013). To confirm the deficiency, AGAL activity was also measured in leukocytes by a fluorometric assay following the procedure described by Shapira et al. (1989).

\subsection{GLA mutation analysis}

The mutation analyses were performed as described by Pasqualim et al. (2014). Briefly, genomic DNA was extracted from FTA Classic cards (Whatmann, USA) according to the manufacturer's instructions. In all patients the GLA open reading frame (exons 1-7 and their flanking regions) was amplified by PCR, as previously described by Shabbeer et al. (2005), with minor modifications. Each amplicon was purified with EXO-SAP (GE Healthcare Lifesciences, USA) and quantified with Low Mass Reader (Invitrogen, USA).

Forward and reverse sequences were analyzed with the same primers used for PCR amplification in an ABI3500 genetic analyzer and BigDye Terminator v3.1 (Applied Biosystems, USA). The obtained sequences were compared to the one reported on GenBank (accession number X14448.1) and sequence variation was confirmed by repeated PCR amplification and sequencing. Sequence variations were described according to the international mutation nomenclature guidelines as set forth by the Human Genome Variation Society (http://www.hgvs.org/ mutnomen).

Data from the Fabry Database (http://www.fabry-database.org) and Human Genome Mutation Database (http://www.hgmd.cf.ac.uk) were used to define novel GLA sequence variants and their associated phenotype. Each mutation was analyzed to determine the relative conservation of the substituted amino acid by comparison with other vertebrate species. To predict the effect of newly identified mutation we used SIFT, PolyPhen2, MutPred, MutationTaster, NetGene2 and Fabry CEP software.

SIFT predicts whether an amino acid substitution affects protein function based on sequence homology and the physical properties of amino acids ( $\mathrm{Ng}$ and Henikoff, 2003). In this way, if a score is smaller than 0.05 , the corresponding neutral substitutions (NS) are predicted as "damaging", while if it is greater, the NS are predicted as "tolerated". PolyPhen 2 is an automatic tool for prediction based on several features, including the sequence, phylogenetic and structural information characterizing the substitution (Adzhubei et al., 2010). The score of PolyPhen 2 ranges from 0 to 1 , and the corresponding prediction is "probably damaging" (coded as " $\mathrm{D}$ " if the score is greater than 0.85 ); "possibly damaging" (coded as "P" if it is between 0.85 and 0.15 ) and "benign" (coded as "B" if it is smaller than 0.15).
MutPred is a web application tool developed to classify an amino acid substitution as disease-associated or neutral in human. In this way, it can be used to predict the molecular cause of disease. Another application (MutationTaster) integrates information from different biomedical databases and uses established analysis tools to predict evolutionary conservation sites, splice-site changes, loss of protein features and changes that might affect the amount of mRNA within the cell (Schwarz et al., 2010). We also used the NetGene2 server to predict possible splice sites in human GAL gene (Hebsgaard et al., 1996) and, in order to determine if the mutations found were candidates for the treatment with chaperones, the program Fabry CEP.

\section{Results}

\subsection{AGAL enzymatic activity}

The nine Colombian FD patients included in this study had low or undetectable levels of AGAL activity in DBS ( 0.0 to $0.4 \mathrm{nmol} / \mathrm{ml} / \mathrm{h}$ ) and leukocytes ( 0.0 to $0.4 \mathrm{nmol} /$ protein $\mathrm{mg} / \mathrm{h}$ ) (Table 1 ), showing an AGAL residual activity ranging from 0.0 to $0.6 \%$.

\subsection{GLA mutations}

The sequence analysis of the complete coding region of GLA in the nine evaluated patients revealed six previously described mutations: c.1024C > T (p.Arg342*), c.804-2_-3delCA (IVS5-2_-3delCA), c.195-1G > C (IVS1-1G > C), c.1072_1074delGAG (p.Glu358del), c.334C > A (p.Arg112Ser) and c.1072G > A (p.E358K) (Table 1). Patients FD-2 and FD-8 displayed two novel variants: c.1051_1052delGT (p.Val351SerfsX23) and c.806G > T (p.Val269Gly) (Fig. 1A and B). The mutations and enzymatic activities are summarized in Fig. 2.

At the protein level, comparative in silico analysis of the novel c.806G > T mutation (p.Val269Gly) revealed a strict conservation of valine at position 269 among vertebrate species. Polyphen 2 predicted that this missense mutation is probably damaging with a score of 0.999, and SIFT software showed probabilistic scores compatible with a potential deleterious effect (p.Val269Gly $=0,0$ ). For the c.1051_1052delGT variant the MutationTaster software predicted a pathogenic effect with a changed splice site and a truncated protein, while NetGene2 predicted that an acceptor splice site would be lost with this mutation.

\subsection{Clinical correlation}

All the patients showed a classical FD phenotype (Table 2). In relation to the patients who showed new variants, patient FD-2 was a 50 -year-old male who had experienced intermittent neuropathic pain in the lower extremities since 6 years of age, fever crisis, heat intolerance and hypohidrosis. Later, when he turned 33 , he showed an increase in proteinuria that quickly progressed to an end-stage renal disease and kidney transplantation.

Patient FD-8 was a 51-years-old male who presented with angiokeratoma at age 6 , and suffered from acroparesthesias, fever crisis, hypohidrosis and heat intolerance from the age of 8.

At the age of 30 he showed increases in proteinuria and developed chronic renal failure.

\section{Discussion}

This is the first study carried out in Colombia that evaluates the molecular aspects of FD. The mutation analysis of the GLA gene in nine FD Colombian patients allowed us to identify eight mutations, six of them previously described and two new variants, illustrating the genetic heterogeneity underlying this disease at the molecular level. 
Table 1

Mutations found in nine Colombian patients with Fabry disease. FD: Fabry disease patient. NA: not available.

\begin{tabular}{|c|c|c|c|c|c|c|c|c|}
\hline \multirow[t]{2}{*}{ Proband } & \multirow[t]{2}{*}{ Phenotype } & \multicolumn{2}{|c|}{ GLA activity } & \multirow[t]{2}{*}{ Mutation site } & \multirow[t]{2}{*}{ Nucleotide change } & \multirow[t]{2}{*}{ Amino acid change } & \multirow[t]{2}{*}{ Mutation type } & \multirow[t]{2}{*}{ Reference } \\
\hline & & DBS & Leukocyte & & & & & \\
\hline FD-1 & Classical & 0.0 & 0.1 & Exon 7 & c. $1024 \mathrm{C}>\mathrm{T}$ & p.Arg342* & Missense & (Davies et al., 1993b) \\
\hline FD-2 & Classical & 0.1 & 0.0 & Exon 6 & c. $806 \mathrm{G}>\mathrm{T}$ & p.Val269Gly & Missense & This study \\
\hline FD-3 & Classical & 0.2 & 0.4 & Intron 5 & c.804-2_-3delCA (IVS5-2_-3delCA) & - & Aberrant splicing & (Eng et al., 1993) \\
\hline FD-4 & Classical & 0.2 & 0.3 & Intron 1 & c.195-1G>C (IVS1-1G>C) & - & Aberrant splicing & (Chen et al., 1998) \\
\hline FD-5 & Classical & 0.4 & NA & Exon 7 & c.1072_1074delGAG & p.Glu358del & Deletion & (Blanch et al., 1996) \\
\hline FD-6 & Classical & 0.1 & 0.3 & Intron 1 & c.195-1G>C (IVS1-1G>C) & - & Aberrant splicing & (Chen et al., 1998) \\
\hline FD-7 & Classical & 0.1 & 0.1 & Exon 7 & c. $1072 \mathrm{G}>\mathrm{A}$ & p.Glu358Lys & Missense & Miyazaki et al., 1998 \\
\hline FD-8 & Classical & 0.2 & 0.4 & Exon 7 & c.1051_1052delGT & p.Val351SerfsX23 & Deletion & This study \\
\hline FD-9 & Classical & 0.02 & NA & Exon 2 & c. $334 \mathrm{C}>\mathrm{A}$ & p.Arg112Ser & Missense & (Ishii et al., 1992) \\
\hline
\end{tabular}

NA $=$ Not available.

We identified a novel mutation in patient FD-8: c.1051_1052delGT (p.Val351SerfsX23). This deletion causes a frame shift in the open reading frame and creates a premature termination codon at residue 373 , probably leading to the degradation of the protein. The c.1051_1052delGT

A

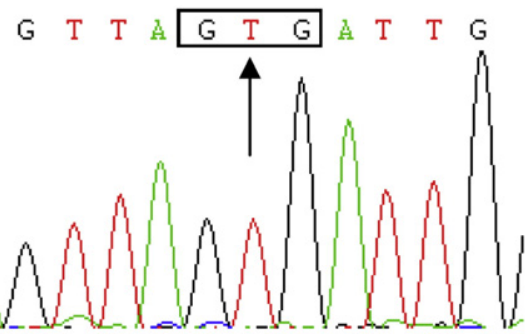

FD-2

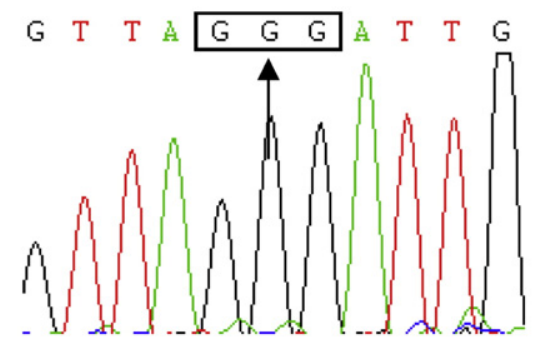

B
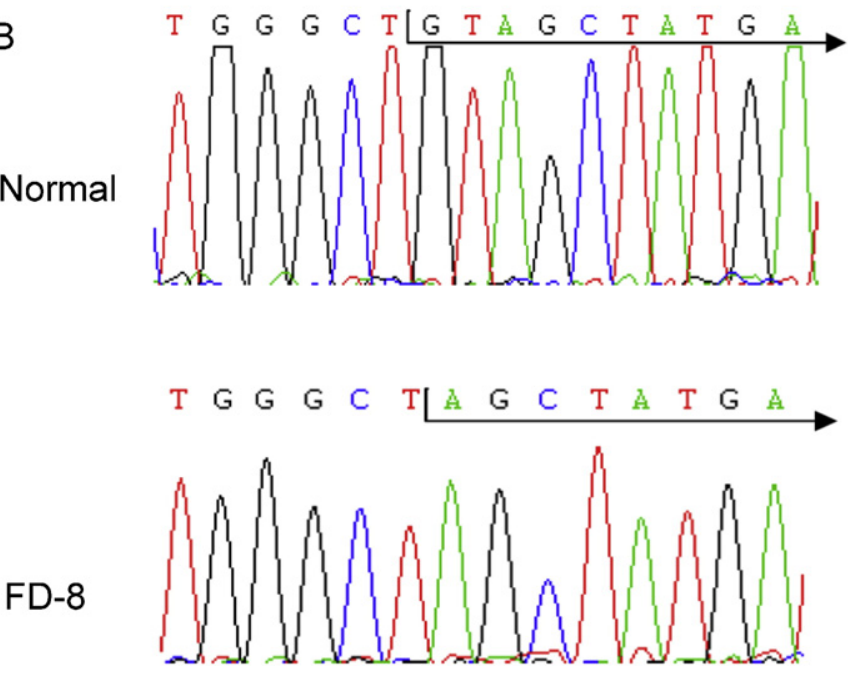

Fig. 1. A. Partial genomic DNA sequence of GLA gene, indicating the single-base substitution of $\mathrm{G}$ to T (c.806G > T; p.Val269Gly) in the hemizygous patient FD-2. B. Partial genomic DNA sequence of GLA gene, indicating the deletion of two nucleotides (c.1051_1052delGT; p.V351SFsX23) in the hemizygous patient FD-8. mutation is characterized by an almost complete lack of GLA activity in the patient and a classical phenotype of FD.

Another novel mutation c.806G > T (p.Val269Gly) in exon 6 was identified in patient FD-2. The substitution of valine for glycine results in a change in the small hydrophobic pocket of the protein, probably leading to misfolding and impairment of substrate binding. Davies et al. (1993a) and Shabbeer et al. (2006) have previously reported different missense mutations (p.Val269Ala; p.Val269Met, respectively) at the same amino acid location in patients with Fabry disease. In these reports the patients also showed a classical FD phenotype, but no more clinical information is given (Davies et al., 1993a; Shabbeer et al., 2006). SIFT and Polyphen2 bioinformatic tools predicted a potential deleterious effect of the p.Val269Gly mutation, thus reinforcing the relation genotype-phenotype. However, it was not possible to perform functional studies on the newly identified mutations, therefore we cannot predict the true implications of this variant in the function of the protein.

The genetic analysis of FD-5 revealed a deletion of $3 \mathrm{bp}$ from nucleotides 1072-1074 in exon 7, resulting in the removal of a glutamic acid codon at residue 358 . This amino acid has an ion pair linkage to lysine and hydrogen bonds to tryptophan. Patient FD-7 had a similar kind of mutation in the residue 358, where the wildtype glutamine changes to lysine: c.1072G > A (p.E358K). Given that charged residues in ion pairs promote the proper folding of proteins, a mutation of one-half of an ion pair introduces an unpaired charge that is energetically highly unfavorable in the interior of the protein, leading to a destabilization of protein's folded conformation (Blanch et al., 1996; Miyazaki et al., 1998).

Patient FD-3 had a deletion of 2 nucleotides $(-2$ and -3$)$ in the acceptor splice site of intron 5 of the GLA gene that disrupted the 3 ' acceptor splice site of intron 5 . This mutation gives rise to a rare aberrant splicing variation (simultaneous $3^{\prime}$ destruction and $3^{\prime}$ creation) and then introduced a termination signal and deletion of 162 residues. This mutation (IVS5-2_-3delCA) had already been described in an Irish patient with classic Fabry disease (Eng et al., 1993), but no correlation genotype/phenotype has been described for it.

The c.195-1G > C (IVS1-1G > C) was the most frequent mutation, found in two presumably unrelated families with FD (FD-4 and FD-6). This mutation was previously reported in Japanese classical hemizygotes with FD and provokes aberrant pre-mRNA splicing. This abnormally processed RNA is subjected to accelerated degradation, resulting in a marked decrease in the amount of mRNA (Okumiya et al., 1996).

Most mutations causing FD are private, occurring in a single or few families even though several mutations at CpG dinucleotides (known mutational hotspots for the disease) occur more often in unrelated families. In this study we found p.Arg112Ser, and p.Arg $342^{*}$ mutations, which have been previously reported in unrelated classically affected families (Shabbeer et al., 2006). Arginine-112 is located on the $\alpha 2$ helix of the $N$-terminal $\beta / \alpha$-barrel, suggesting that a substitution to serine is predicted to destabilize the disulfide bond and the pocket that the arginine occupies, leading to protein misfolding (Shabbeer 


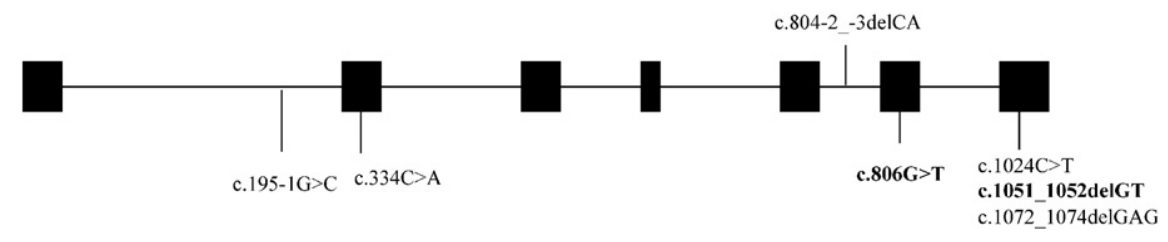

Fig. 2. Map of the GLA gene with alterations found in Colombian patients with FD.

et al., 2006). Meanwhile, the p.Arg342* mutation will result in the generation of a truncated protein lacking exon 7 , which has been known to be crucial for normal enzymatic function (Ishii et al., 1994, 1995; Miyamura et al., 1996).

The mutation profile here described is consistent with the classical disease presentation and the complete lack of AGAL enzyme activity seen in all the analyzed patients. When comparing the mutations present in the Colombian population with the mutation profile identified in other Latin American countries (Argentina, Mexico and Brazil) (Politei et al., 2013; Ramos-Kuri et al., 2014; Rozenfeld et al., 2006) and Spain (Pereira et al., 2007; Turaça et al., 2012; Rodríguez-Marí et al., 2003) no association is found. This is probably a consequence of the limited sample size analyzed in most of the previous studies that evaluate the mutation profile, such as Argentinean and Mexican studies. Our results are consistent with the correlation between the presence of splicing site mutations and the manifestation of a classical FD phenotype previously described by Saito et al. (2013), since all the patients bearing this type of mutation showed a classical phenotype.

The molecular analysis is an important diagnostic tool to determine the possible benefits of treatment with therapeutic strategies such as pharmacological chaperones. Given that this therapy can only be effective in patients with misfolding mutations, the decision of using it depends on an adequate genotyping based on a case-by-case scenario (Andreotti et al., 2010). In order to see which of these patients would be candidates for this kind of therapy we used the Fabry_CEP program.
According to the results obtained, only patients bearing two of the eight mutations found (p.Val269Gly and p.Arg112Ser) had a probability of $59 \%$ to respond to this therapy.

In summary, this work intends to make an important contribution for the understanding of the molecular basis of FD in Colombia. Here we showed that there is a high variability in the mutations leading to disease, which highlights the importance of the identification of mutations in the GLA gene for the diagnosis, carrier identification, genetic counseling and use of new therapeutic strategies (such as chaperone therapy). Given that most mutations in patients with FD are private, it is necessary to use molecular analyses as a diagnostic tool in order to offer a wider spectrum of therapeutic options for FD patients and families.

\section{Conclusions}

- Six previously described mutations were found and two new ones are described for the Colombian population.

- There is a high variability of mutations in Colombian patients with Fabry disease.

- A small correlation between the predicted consequence of the mutation in the protein and the classical FD phenotype was found.

- The prevalence of the mutations here described, differs from what has been reported in other Latin American countries and Spain.

- Only two of the eight detected mutations seem to be candidates,

Table 2

Clinical and molecular characteristic of Colombian patients with Fabry Disease.

\begin{tabular}{|c|c|c|c|c|c|c|c|c|c|}
\hline Patient & FD-1 & FD-2 & FD-3 & FD-4 & FD-5 & FD-6 & FD-7 & FD-8 & FD-9 \\
\hline Mutation & c. $1024 \mathrm{C}>\mathrm{T}$ & c. $806 \mathrm{G}>\mathrm{T}$ & c.804-2_-3delCA & c. $195-1 G>C$ & c.1072_1074delGAG & c. $195-1 G>C$ & c. $1072 \mathrm{G}>\mathrm{A}$ & c.1051_1052delGT & c. $334 \mathrm{C}>\mathrm{A}$ \\
\hline \multicolumn{10}{|l|}{ Symptoms } \\
\hline \multicolumn{10}{|l|}{ Neurologic } \\
\hline Neuropathic Pain & + & - & + & + & - & + & + & + & - \\
\hline Acroparesthesia & + & + & + & + & - & + & + & + & - \\
\hline Heat or cold intolerance & + & + & + & + & - & + & + & + & + \\
\hline Fever Crisis & + & + & + & - & - & - & + & + & - \\
\hline Strokes & - & - & - & + & - & - & + & - & - \\
\hline Seizures & - & - & - & - & - & - & - & - & - \\
\hline \multicolumn{10}{|l|}{ Tegumentary } \\
\hline Angiokeratoma & + & - & + & + & - & + & + & + & + \\
\hline Hypohydrosis & + & - & + & + & - & + & + & + & + \\
\hline \multicolumn{10}{|l|}{ Kidney } \\
\hline Renal Failure & - & - & + & + & + & + & + & + & - \\
\hline Dialysis & - & - & + & - & + & - & - & - & - \\
\hline Hematuria & + & - & - & + & + & + & + & - & - \\
\hline Proteinuria & + & + & + & + & + & + & - & + & + \\
\hline \multicolumn{10}{|l|}{ Cardiovascular } \\
\hline Chest Pain & + & - & - & + & - & - & - & - & - \\
\hline Dyspnea & + & - & - & + & - & - & - & - & - \\
\hline Syncope & - & - & - & + & - & - & - & - & - \\
\hline Hypertension & + & - & - & + & - & - & + & - & - \\
\hline Left ventricular hypertrophy & + & - & - & + & - & - & - & - & - \\
\hline \multicolumn{10}{|l|}{ Ophthalmologic } \\
\hline Corneal opacities & + & - & - & + & - & - & - & - & - \\
\hline \multicolumn{10}{|l|}{ Gastrointestinal } \\
\hline Nauseas & + & - & - & - & - & - & - & - & - \\
\hline Abdominal Pain & + & + & - & + & - & + & + & - & + \\
\hline Chronic diarrhea & + & - & - & + & - & - & - & - & - \\
\hline \multicolumn{10}{|l|}{ Metabolic } \\
\hline Dyslipidemia & - & - & - & + & - & + & - & - & - \\
\hline
\end{tabular}


with a $59 \%$ probability, for new therapeutic treatments such as chaperone therapy.

\section{Compliance with ethical standards}

\section{Conflict of interest:}

Alfredo Uribe, Heidi Eliana Mateus, Juan Carlos Prieto, Maria Fernanda Palacios, Sandra Yaneth Ospina, Gabriela Pasqualim, Ursula da Silveira Matte and Roberto Giugliani declare that they have no conflict of interest.

Informed consent:

All procedures followed were in accordance with the ethical standards of the responsible committee on human experimentation (institutional and national) and with the Helsinki Declaration of 1975, as revised in 2000 (5). Informed consent was obtained from all patients included in the study.

\section{Acknowledgments}

This work was supported by the Universidad del Rosario, Pontificia Universidad Javeriana, Universidad de los Andes and by Genzyme Corporation Colombia. We would like to thank Natalia Pacheco for her help in editing this paper.

\section{References}

Adzhubei, I.A., Schmidt, S., Peshkin, L., Ramensky, V.E., Gerasimova, A., Bork, P., Kondrashov, A.S., Sunyaev, S.R., 2010. A method and server for predicting damaging missense mutations. Nat. Methods 7, 248-249.

Andreotti, G., Guarracino, M.R., Cammisa, M., Correra, A., Cubellis, M.V., 2010. Prediction of the responsiveness to pharmacological chaperones: lysosomal human alphagalactosidase, a case of study. Orphanet J. Rare Dis. 5, 36.

Ashley, G.A., Shabbeer, J., Yasuda, M., Eng, C.M., Desnick, R.J., 2001. Fabry disease: twenty novel alpha-galactosidase A mutations causing the classical phenotype. J. Hum. Genet. 46, 192-196.

Barker, D., Schafer, M., White, R., 1984. Restriction sites containing CpG show a higher frequency of polymorphism in human DNA. Cell 36, 131-138.

Blanch, L.C., Meaney, C., Morris, C.P., 1996. A sensitive mutation screening strategy for Fabry disease: detection of nine mutations in the alpha-galactosidase A gene. Hum. Mutat. 8, 38-43.

Brady, R.O., 1967. Enzymatic abnormalities in diseases of sphingolipid metabolism. Clin. Chem. 13, 565-577.

Chamoles, N.A., Blanco, M., Gaggioli, D., 2001. Fabry disease: enzymatic diagnosis in dried blood spots on filter paper. Clin. Chim. Acta 308, 195-196.

Chen, C.H., Shyu, P.W., Wu, S.J., Sheu, S.S., Desnick, R.J., Hsiao, K.J., 1998. Identification of a novel point mutation (S65T) in alpha-galactosidase A gene in Chinese patients with Fabry disease. Mutations in brief no. 169. Online. Hum Mutat 11, 328-330.

Civallero, G., Michelin, K., de Mari, J., Viapiana, M., Burin, M., Coelho, J.C., Giugliani, R., 2006. Twelve different enzyme assays on dried-blood filter paper samples for detection of patients with selected inherited lysosomal storage diseases. Clin. Chim. Acta 372, 98-102.

Cooper, D.N., Youssoufian, H., 1988. The CpG dinucleotide and human genetic disease Hum. Genet. 78, 151-155.

Davies, J.P., Winchester, B.G., Malcolm, S., 1993a. Mutation analysis in patients with the typical form of Anderson-Fabry disease. Hum. Mol. Genet. 2, 1051-1053.

Davies, J.P., Winchester, B.G., Malcolm, S., 1993b. Sequence variations in the first exon of alpha-galactosidase A. J Med Genet 30, 658-663.

Desnick, R.J., Brady, R.O., 2004. Fabry disease in childhood. J. Pediatr. 144, S20-S26.

Desnick, R.J., Brady, R., Barranger, J., Collins, A.J., Germain, D.P., Goldman, M., Grabowski, G., Packman, S., Wilcox, W.R., 2003. Fabry disease, an under-recognized multisystemic disorder: expert recommendations for diagnosis, management, and enzyme replacement therapy. Ann. Intern. Med. 138, 338-346.

Eng, C.M., Resnick-Silverman, L.A., Niehaus, D.J., Astrin, K.H., Desnick, R.J., 1993. Nature and frequency of mutations in the alpha-galactosidase A gene that cause Fabry disease. Am. J. Hum. Genet. 53, 1186-1197.

Hebsgaard, S.M., Korning, P.G., Tolstrup, N., Engelbrecht, J., Rouzé, P., Brunak, S., 1996. Splice site prediction in Arabidopsis thaliana pre-mRNA by combining local and global sequence information. Nucleic Acids Res. 24, 3439-3452.

Ishii, S., Sakuraba, H., Suzuki, Y., 1992. Point mutations in the upstream region of the alpha-galactosidase A gene exon 6 in an atypical variant of Fabry disease. Hum Genet 89, 29-32.

Ishii, S., Kase, R., Sakuraba, H., Fujita, S., Sugimoto, M., Tomita, K., Semba, T., Suzuki, Y. 1994. Human alpha-galactosidase gene expression: significance of two peptide regions encoded by exons 1-2 and 6. Biochim. Biophys. Acta 1204, 265-270.

Ishii, S., Kase, R., Sakuraba, H., Suzuki, Y., 1995. The functional role of glutamine-280 and threonine-282 in human alpha-galactosidase. Biochim. Biophys. Acta 1270, 163-167.
Lin, H.Y., Chong, K.W., Hsu, J.H., Yu, H.C., Shih, C.C., Huang, C.H., Lin, S.J., Chen, C.H., Chiang, C.C., Ho, H.J., Lee, P.C., Kao, C.H., Cheng, K.H., Hsueh, C., Niu, D.M., 2009. High incidence of the cardiac variant of Fabry disease revealed by newborn screening in the Taiwan Chinese population. Circ. Cardiovasc. Genet. 2, 450-456.

Lukas, J., Giese, A.K., Markoff, A., Grittner, U., Kolodny, E., Mascher, H., Lackner, K.J., Meyer, W., Wree, P., Saviouk, V., Rolfs, A., 2013. Functional characterisation of alphagalactosidase a mutations as a basis for a new classification system in Fabry disease. PLoS Genet. 9, e1003632.

Meikle, P.J., Grasby, D.J., Dean, C.J., Lang, D.L., Bockmann, M., Whittle, A.M., Fietz, M.J., Simonsen, H., Fuller, M., Brooks, D.A., Hopwood, J.J., 2006. Newborn screening for lysosomal storage disorders. Mol. Genet. Metab. 88, 307-314.

Miyamura, N., Araki, E., Matsuda, K., Yoshimura, R., Furukawa, N., Tsuruzoe, K., Shirotani, T., Kishikawa, H., Yamaguchi, K., Shichiri, M., 1996. A carboxy-terminal truncation of human alpha-galactosidase $A$ in a heterozygous female with Fabry disease and modification of the enzymatic activity by the carboxy-terminal domain. Increased, reduced, or absent enzyme activity depending on number of amino acid residues deleted. J. Clin. Invest. 98, 1809-1817.

Miyazaki, T., Kajita, M., Ohmori, S., Mizutani, N., Niwa, T., Murata, Y., Seo, H., 1998. A novel mutation (E358K) in the alpha-galactosidase A gene detected in a Japanese family with Fabry disease. Hum. Mutat. Suppl. 1, S139-S140.

Nakao, S., Takenaka, T., Maeda, M., Kodama, C., Tanaka, A., Tahara, M., Yoshida, A., Kuriyama, M., Hayashibe, H., Sakuraba, H., 1995. An atypical variant of Fabry's disease in men with left ventricular hypertrophy. N. Engl. J. Med. 333, 288-293.

Nakao, S., Kodama, C., Takenaka, T., Tanaka, A., Yasumoto, Y., Yoshida, A., Kanzaki, T., Enriquez, A.L., Eng, C.M., Tanaka, H., Tei, C., Desnick, R.J., 2003. Fabry disease: detection of undiagnosed hemodialysis patients and identification of a "renal variant" phenotype. Kidney Int. 64, 801-807.

Ng, P.C., Henikoff, S., 2003. SIFT: predicting amino acid changes that affect protein function. Nucleic Acids Res. 31, 3812-3814.

Okumiya, T., Takenaka, T., Ishii, S., Kase, R., Kamei, S., Sakuraba, H., 1996. Two novel mutations in the alpha-galactosidase gene in Japanese classical hemizygotes with Fabry disease. Jpn. J. Hum. Genet. 41, 313-321.

Pasqualim, G., Simon, L., Sperb-Ludwig, F., Burin, M.G., Michelin-Tirelli, K., Giugliani, R., Matte, U., 2014. Fabry disease: a new approach for the screening of females in high-risk groups. Clin Biochem 47, 657-662.

Pereira, F.S., Jardim, L.B., Netto, C.B., Burin, M.G., Cecchin, C., Giugliani, R., Matte, U.S., 2007. Genomic analysis of Brazilian patients with Fabry disease. Braz. J. Med. Biol. Res. 40, 1599-1604.

Politei, J.M., Cabrera, G., Amartino, H., Valdez, R., Masllorens, F., Ripeau, D., Antongiovanni, N., Soliani, A., Luna, P., Cedrolla, M., Fernandez, S., Fainboim, A., 2013. Fabry disease in Argentina: an evaluation of patients enrolled in the Fabry Registry. Int. J. Clin. Pract. 67, 66-72.

Ramos-Kuri, M., Olvera, D., Morales, J.J., Rodriguez-Espino, B.A., Lara-Mejía, A., De Los, Ríos D., Obrador, G.T., Granados, J., Correa-Rotter, R., 2014. Clinical, histological and molecular characteristics of Mexican patients with Fabry disease and significant renal involvement. Arch. Med. Res. 45, 257-262.

Rodríguez-Marí, A., Coll, M.J., Chabás, A., 2003. Molecular analysis in Fabry disease in Spain: fifteen novel GLA mutations and identification of a homozygous female. Hum. Mutat. 22, 258.

Rozenfeld, P.A., Tarabuso, A., Ebner, R., Ramallo, G., Fossati, C.A., 2006. A successful approach for the detection of Fabry patients in Argentina. Clin. Genet. 69, 344-348.

Saito, S., Ohno, K., Sakuraba, H., 2013. Comparative study of structural changes caused by different substitutions at the same residue on $\alpha$-galactosidase A. PLoS One 8, e84267.

Schirinzi, A., Centra, M., Prattichizzo, C., Gigante, M., De Fabritiis, M., Giancaspro, V., Petrarulo, F., Santacroce, R., Margaglione, M., Gesualdo, L., Ranieri, E., 2008. Identification of GLA gene deletions in Fabry patients by Multiplex Ligation-dependent Probe Amplification (MLPA). Mol. Genet. Metab. 94, 382-385.

Schwarz, J.M., Rödelsperger, C., Schuelke, M., Seelow, D., 2010. MutationTaster evaluates disease-causing potential of sequence alterations. Nat. Methods 7, 575-576.

Shabbeer, J., Robinson, M., Desnick, R.J., 2005. Detection of alpha-galactosidase a mutations causing Fabry disease by denaturing high performance liquid chromatography. Hum Mutat 25, 299-305.

Shabbeer, J., Yasuda, M., Benson, S.D., Desnick, R.J., 2006. Fabry disease: identification of 50 novel alpha-galactosidase A mutations causing the classic phenotype and threedimensional structural analysis of 29 missense mutations. Hum. Genomics 2, 297-309.

Shapira, E., Blitzer, M.G., Africk, D.K., Miller, J.B., 1989. Biochemical Genetics: A Laboratory Manual. First edn. Oxford University Press, New York.

Spada, M., Pagliardini, S., Yasuda, M., Tukel, T., Thiagarajan, G., Sakuraba, H., Ponzone, A., Desnick, R.J., 2006. High incidence of later-onset fabry disease revealed by newborn screening. Am. J. Hum. Genet. 79, 31-40.

Terryn, W., Deschoenmakere, G., De Keyser, J., Meersseman, W., Van Biesen, W., Wuyts, B., Hemelsoet, D., Pascale, H., De Backer, J., De Paepe, A., Poppe, B., Vanholder, R., 2013. Prevalence of Fabry disease in a predominantly hypertensive population with left ventricular hypertrophy. Int J Cardiol 167, 2555-2560.

Turaç, L.T., Pessoa, J.G., Motta, F.L., Muñoz Rojas, M.V., Müller, K.B., Lourenço, C.M., Junior Marques, W., D'Almeida, V., Martins, A.M., Pesquero, J.B., 2012. New mutations in the GLA gene in Brazilian families with Fabry disease. J. Hum. Genet. 57, 347-351.

Uribe, A., Giugliani, R., 2013. Selective screening for lysosomal storage diseases with dried blood spots collected on filter paper in 4,700 high-risk colombian subjects. JIMD Rep. 11, 107-116.

Yoshimitsu, M., Higuchi, K., Miyata, M., Devine, S., Mattman, A., Sirrs, S., Medin, J.A., Tei, C., Takenaka, T., 2011. Identification of novel mutations in the $\alpha$-galactosidase A gene in patients with Fabry disease: pitfalls of mutation analyses in patients with low $\alpha$ galactosidase A activity. J. Cardiol. 57, 345-353. 